\title{
PIEZOELECTRIC FORCE SENSORS FOR HEXAPOD TRANSPORTATION PLATFORM
}

\author{
Mindaugas Luneckas ${ }^{1}$, Tomas Luneckas ${ }^{2}$, Vytenis Gavelis ${ }^{3}$, Vytautas Valaitis ${ }^{4}$, Dainius Udris ${ }^{5}$ \\ ${ }^{1,2,5}$ Dept of Automation, Vilnius Gediminas Technical University, Lithuania \\ ${ }^{3}$ Dept of Electronics Engineering, Kaunas University of Technology, Lithuania \\ ${ }^{4}$ Dept of Software Engineering, Vilnius University, Lithuania
}

Submitted 29 November 2014; resubmitted 10 May 2015, 18 June 2015; accepted 25 June 2015

\begin{abstract}
Rough terrain is one of the major issues for transporting various objects to different remote locations. Wheeled platforms or robots are not suitable for such tasks due to a lack of ground clearance. Walking robots, despite their slower speed, can be successfully used as transportation platforms that can overcome the environment. However, leg placing requires accurate supervision and the force sensing system must be developed on each foot to acquire equal force distribution between legs and to obtain stable motion over the irregular surface. In this paper, we investigate the improvement of the hexapod robot's feet by upgrading them with piezoelectric force sensors. By monitoring force dependence on transferred legs, we establish the most suitable hexapod gait for moving over the even surface.
\end{abstract}

Keywords: hexapod robot; piezoelectric sensors; transportation platform; hexapod gait.

\section{Introduction}

Payload transportation in space missions or any earthly task (Ettlin, Bleuler 2006), e.g. underground mining operations, catastrophic territories, crossing bridges or any fragile surfaces, are considered a significantly difficult task. Each of the mentioned situations requires either rough terrain traversability or a very stable and careful movement. All wheeled robots can only overcome roughness that is smaller than the radius of their wheel. Due to this problem, the use of all wheeled (also tracked) platforms is limited. That is why walking robots have been attracting a lot of attention over the last few years (Pongas et al. 2007).

However, achieving a complete and perfect legged locomotion over the rough terrain is a very complex task. Special kinematic models and sensors must be used to obtain the necessary movement. A large amount of work concerning the rough terrain traversability was done.

There are three main reasons why force sensing is used: to adapt to the roughness of terrain, to equally distribute forces between feet (Schmucker et al. 2005) and to know the exact moment of a foot reaching the ground. Otherwise, it is impossible to control the foot pressure against the ground, which might damage the robot, surface or a fragile cargo. A good example could be the ex- periment made with a robotic foot and its interaction with a terrain (Ding et al. 2013). The experiment was made using a robotic foot constructed separately from the whole robot. Force distribution was calculated theoretically and tested experimentally, but no sensors were used. Three different categories were distinguished: hard foot on deformable terrain, deformable foot on hard terrain and deformable foot on the deformable terrain. Results clearly showed that foot placement deforms either the foot or the terrain, depending on softness of the material. But without force sensors it is impossible to develop a good algorithm that would not allow the robot to press feet hard against the surface. Also, nothing was mentioned about the possible adaptability to irregular terrain.

Another work concerning rough terrain traversability is described in detail in (Kalakrishnan et al. 2009). Terrain templates (different height maps under foothold) make it easier to adapt to a previously unseen rough terrain. Although the results are promising, and the robot can successfully overcome the rough terrain, nothing is mentioned about sensing or force distribution between the feet. It still remains unknown, whether or not the robot damages the surface or its legs. More robust and compliant locomotion was acquired by Buchli et al. (2009) using force sensors. Experiments were carried out on the irregular terrain and results showed that 
a robot with force sensors can surpass irregular terrain faster. Still, no results were given about the actual size of the force in each foot.

\section{Piezoelectric Sensors}

There are many different sensors that could be used as robot's force sensing: barometers, pressure sensors, tactile sensors, load cells or silicon-based sensors. In this work, we chose to upgrade hexapod robot's feet with piezoelectric sensors (Fig. 1). Unlike silicon-based sensors that are relatively small and brake in case of an overload (Beebe et al. 1998), piezoelectric sensors are of needed size and can withstand high pressure.

Main advantages of these sensors are:

- low cost;

- high resolution (deflections can be micrometre size);

- wide measuring range;

- signal can be easily reproduced;

- high temperature resistance;

- insensitive to external electric and magnetic fields.

Another reason we chose to use piezoelectric sensors is that they can be easily placed on robot's feet (Fig. 2). Not to break sensors or wires, we glued the hemisphere at the bottom and a small metal plate at the top. The whole robot with piezoelectric sensors is shown in Fig. 3.

As we didn't have the characteristics of piezoelectric sensors, the experiment was made using an oscilloscope to obtain the voltage dependence on the pressure force $U=f(F)$. We performed the experiment taking into account the parameters of robot movement so that the characteristic would be as much useful as possible. Results of the experiment are shown in Fig. 4.

Looking at visible points in Fig. 4, it appears that voltage dependence on pressure force is linear:

$$
U=1.04 \cdot F+4.78
$$

Following experimental results on the hexapod robot, the current diagrams will be recalculated by above mentioned equation. Though dependence is not very accurate because of big measurement data scatter, the result is adequate to perform our experiment. To increase force sensing accuracy, a more accurate characteristic for the piezoelectric sensor should be obtained.

\section{Experimental Setup}

For our experiment, we used three most common hexapod gaits: tripod, tetrapod and wave gait (Fig. 5). In each case, we monitored all transfer legs and one additional support leg using a four-channel oscilloscope. Abbreviations of each leg are described in Table 1.

It is of great importance to point out the RM leg, which was used in all cases. By monitoring the additional support leg, it becomes possible to distinguish the start and end of each gait. Also, it is much easier to see the exact moment the robot raises and places its legs on the surface.

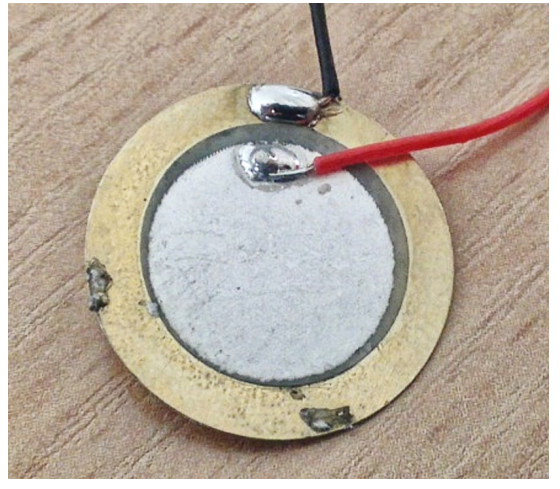

Fig. 1. Piezoelectric sensors

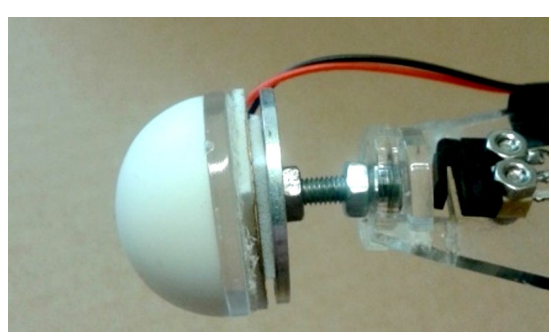

Fig. 2. Foot of a hexapod robot with an integrated piezoelectric sensor

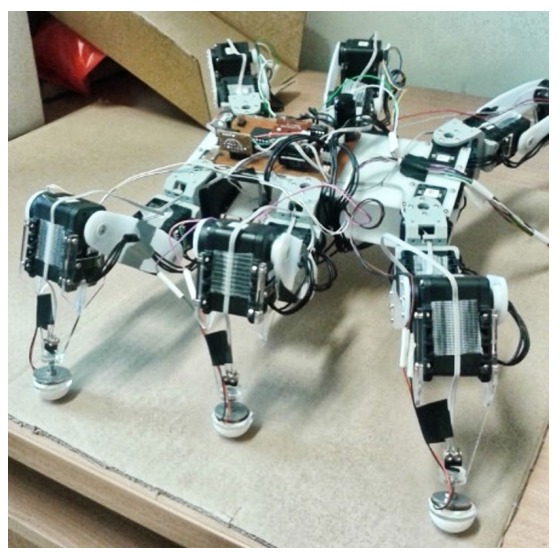

Fig. 3. Hexapod robot upgraded with piezoelectric sensors

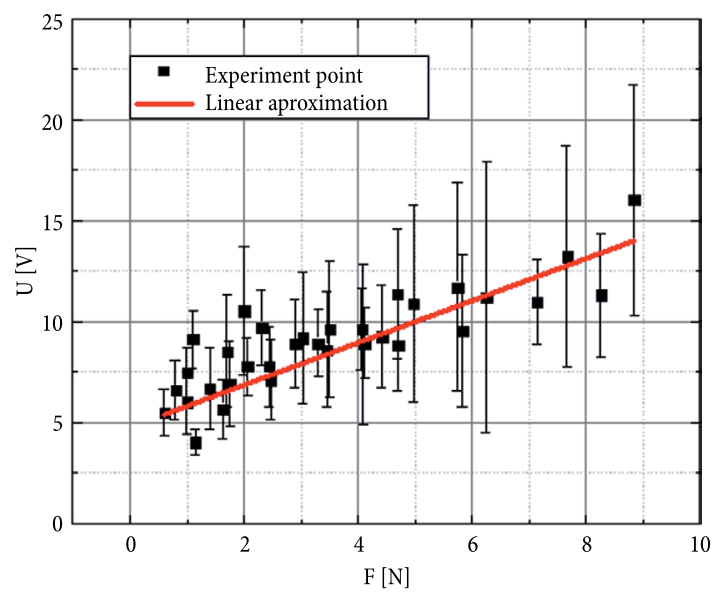

Fig. 4. Experimental characteristics of piezoelectric sensors $U=f(F)$ 
Table 1. Leg abbreviations

\begin{tabular}{ccl}
\hline Abbreviation & Name of the leg & \multicolumn{1}{c}{ Comment } \\
\hline RF & Right Front & Used during tripod and tetrapod gait experiments \\
\hline RM & Right Middle & $\begin{array}{l}\text { Used in all experiments as additional supporting leg, which is always positioned } \\
\text { on ground while transfer legs are in swinging motion }\end{array}$ \\
\hline RH & Right Hind & Used only during the tripod gait experiment \\
\hline LF & Left Front & Were not used in any of the experiments \\
\hline LM & Left Middle & Used in all experiments \\
\hline LH & Left Hind & Were not used in any of the experiments \\
\hline
\end{tabular}

a)

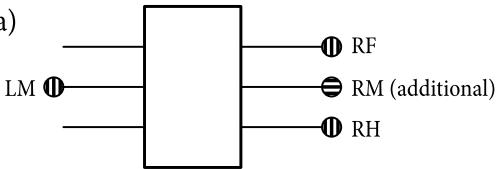

b)
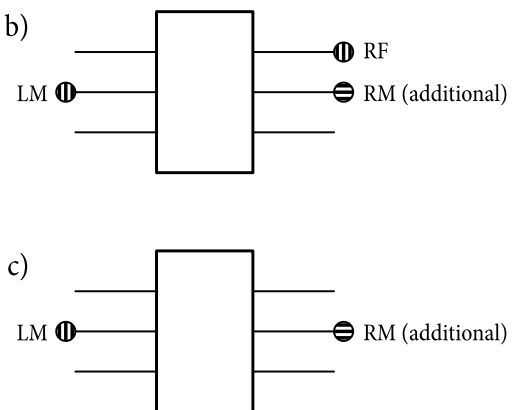

Fig. 5. Robot legs that were monitored during each experiment for: $\mathrm{a}$ - tripod gait; $\mathrm{b}$ - tetrapod gait;

$$
\text { c - wave gait }
$$

\section{Results}

Fig. 6 gives the voltage time diagrams for tripod and wave gaits of the hexapod robot. A smooth filter was added with polynomial order of 2 to distinguish the unwanted noise. As we can see, there is an exponential decrease in voltage. The decrease can be explained by piezoelectric sensors reducing the force to zero following an impact. In our case, only the voltage peaks matter, so lower cut-off frequency is not important. All voltage and recalculated force peaks are presented in Table 2 . Given values were measured at the moment of collision between the feet and ground. It is obvious that when the robot is moving using the tripod gait, legs are pressed against the surface with the least force. This is because the force is distributed between three feet; and when using the wave gait, the robot legs are pressed with the most force because only one leg is pressed against the surface.

Table 2. Force peak values for different gaits

\begin{tabular}{l|lllll|llll|lll|l}
\hline \multicolumn{1}{c|}{ Gait } & \multicolumn{4}{|c|}{ Tripod } & \multicolumn{3}{c|}{ Tetrapod } & \multicolumn{2}{c}{ Wave } \\
\hline $\begin{array}{l}\text { Robot's } \\
\text { leg }\end{array}$ & RF & LM & RH & RM & RF & LM & RM & LM & RM \\
\hline$F[\mathrm{~N}]$ & 0.28 & 2.75 & 1.72 & 2.17 & 0.38 & 3.06 & 3.93 & 3.78 & 5.47 \\
\hline
\end{tabular}

It is also noticeable that voltage time diagrams of the tripod gait have fewer fluctuations than those of the wave gait. This is mainly because the wave gait has six phases, and the tripod gait has only two. In our case, the more phases a gait has, the faster legs are transferred.

Also, Fig. 6 shows negative voltage values. This is because piezoelectric sensors are sensitive in both directions. It means that after the first impact, we have positive values. After the release of pressure, we have deformation toward the opposite direction, thus values become negative. This way, we can distinguish the moment the robot raises its legs (negative peaks) and the moment of impact with the ground (positive peaks). a)

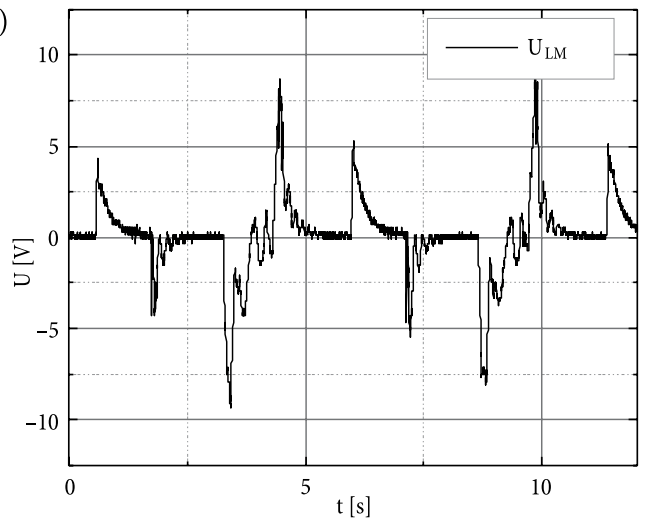

b)

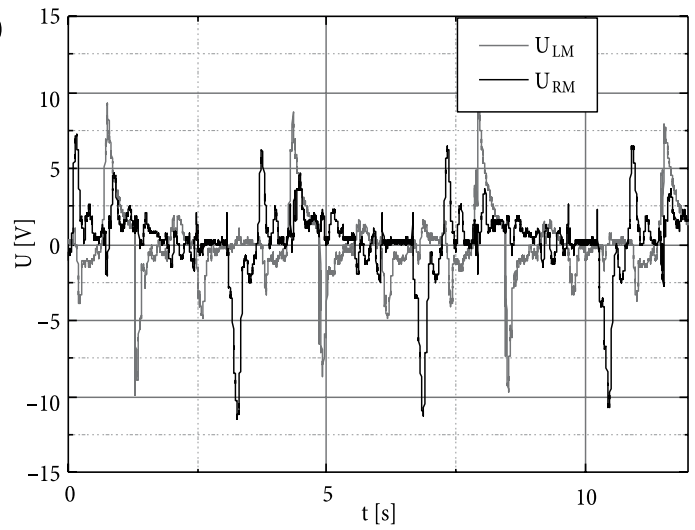

Fig. 6. Voltage time diagrams for: $\mathrm{a}$ - tripod gait; $\mathrm{b}$ - wave gait 


\section{Conclusions}

This paper discusses the dependence of the force distribution on different gaits. Tripod, tetrapod and wave gaits were used to monitor the actual size of the force on each foot during the impact with the ground. Experiments were carried out on the even terrain. Received results clearly demonstrate that during the tripod gait, the robot's feet were pressed against the ground with the least force. And during the wave gait, the robot pressed its feet with the most force. This can be clarified as the force distribution between transfer feet. The distribution occurs during the moment of impact with the surface. The more legs are pressed against the ground, the lesser is the force.

Based on the current results, one of the following solutions should be used for the hexapod robot to eliminate feet pressure:

- a special leg placement algorithm could be developed to slow down the leg speed before the moment of collision with the surface;

- the robot's program could be upgraded with a force indicator, which would not allow the robot to press the feet harder than the given force; although, this method requires a different type of force sensors.

Piezoelectric sensors are used only to monitor the impact forces. After the impact, the force is exponentially reduced to zero again. It is only possible to observe the moment of collision. Also, when working on piezoelectric characteristics, we encountered additional problems. Verification of the appropriate voltage dependence on the force is a matter of another topic due to the complexity of experiment and dependence on various parameters (temperature, the point of impact, acceleration). The future aim is to monitor the force distribution for a longer period. We intend to build unique barometer sensors for the hexapod robot. Also, it will be possible to observe the dependence of the force and energy consumption with different loads. This will give us information about the size of the maximum weight the robot can carry to decide on operations, for which it could be used.

\section{References}

Beebe, D. J.; Denton, D. D.; Radwin, R.G.; Webster, J. G. 1998. A silicon-based tactile sensor for finger-mounted applications, IEEE Transactions on Biomedical Engineering 45(2): 151-159. http://dx.doi.org/10.1109/10.661263

Buchli, J.; Kalakrishnan, M.; Mistry, M.; Pastor, P.; Schaal, S. 2009. Compliant quadruped locomotion over rough terrain, in IEEE/RSJ International Conference on Intelligent Robots and Systems: IROS 2009, 10-15 October 2009, St. Louis, MO, 814-820. http://dx.doi.org/10.1109/IROS.2009.5354681

Ding, L.; Gao, H.; Deng, Z.; Song, J.; Liu, Y.; Liu, G.; Iagnemma, K. 2013. Foot-terrain interaction mechanics for legged robots: modeling and experimental validation, The International Journal of Robotics Research 32(13): 1585-1606. http://dx.doi.org/10.1177/0278364913498122
Ettlin, A.; Bleuler, H. 2006. Rough-terrain robot motion planning based on obstacleness, in 9th International Conference on Control, Automation, Robotics and Vision: ICARCV'06, 5-8 December 2006, Singapore, 1-6. http://dx.doi.org/10.1109/ICARCV.2006.345116

Kalakrishnan, M.; Buchli, J.; Pastor, P.; Schaal, S. 2009. Learning locomotion over rough terrain using terrain templates, in IEEE/RSJ International Conference on Intelligent Robots and Systems: IROS 2009, 10-15 October 2009, St. Louis, MO, 167-172. http://dx.doi.org/10.1109/IROS.2009.5354701

Pongas, D.; Mistry, M.; Schaal, S. 2007. A robust quadruped walking gait for traversing rough terrain, in 2007 IEEE International Conference on Robotics and Automation, 10-14 April 2007, Roma, Italy, 1474-1479. http://dx.doi.org/10.1109/ROBOT.2007.363192

Schmucker, U.; Schneider, A.; Rusin, V.; Zavgorodniy, Y. 2005. Force sensing for walking robots, in Proceedings of the $3 \mathrm{rd}$ International Symposium on Adaptive Motion of Animals and Machines (AMAM2005), 25-30 September 2005, Ilmenau, Germany, 1-6. 